\title{
Signal Transmission with Intra-body and Inter-body Communications
}

This paper was downloaded from TechRxiv (https://www.techrxiv.org).

\section{LICENSE}

CC BY 4.0

SUBMISSION DATE / POSTED DATE

$13-01-2021 / 15-01-2021$

\section{CITATION}

Ahmed, Doaa; Kirchner, Jens; Fischer, Georg (2021): Signal Transmission with Intra-body and Inter-body Communications. TechRxiv. Preprint. https://doi.org/10.36227/techrxiv.13570604.v1

$\mathrm{DOI}$

10.36227/techrxiv.13570604.v1 


\title{
Signal Transmission with Intra-body and Inter-body Communications (Simulation-based Models)
}

\author{
Doaa Ahmed ${ }^{[0000-0001-8356-8811]}$, Jens Kirchner, and Georg \\ Fischer ${ }^{[0000-0002-4033-2005]}$ \\ Institute for Electronics Engineering, Friedrich-Alexander-Universität \\ Erlangen-Nürnberg (FAU), Germany \\ Email: \{doaa.ahmed, jens.kirchner, georg.fischer\}@fau.de
}

\begin{abstract}
In this paper, we investigate two types of data transmission in human body communication (HBC) with galvanic coupling: Intrabody communication along a human arm and inter-body communication between two arms in touch. For the former, the effect of bending the arm is investigated, too. The arms were modeled as five-layers concentric cylinders of different types of tissues. For simulation, the finite element method (FEM) COMSOL Multiphysics5.3a software was used. The influence of different HBC key parameters including applied frequency, distance between transmitter (TX) and receiver (RX), bending, contact area between the contiguous models, and induced current were investigated. The results show that the transmission loss increases with the increase of the transmission length and operating frequency. The electrical potential is directly proportional to the induced current. Bending helps to improve the detected signal in the cases of short distance between TX and RX around the curvature. For distant transceivers, both straight and bended models tend to behave in a close manner. The signal degradation in inter-body communication is considerably higher compared to intra-body communication at the same horizontal distance between TX and RX. At frequencies above $200 \mathrm{kHz}$, both inter-body and intra-body communication give close values when the contact area between the arms covers the distance between TX and RX electrodes. In addition, by increasing the contact area and avoiding gaps between the models, the detected signal is improved. The results illustrate the main determinants of information transmission between both sensors within a body-sensors-network and between different persons.
\end{abstract}

Keywords: Cole-Cole expressions · Dielectric properties of human tissues · Finite Element Model · Galvanic coupling · Human body communication · Inter-body communication $\cdot$ Intra-body communication.

\section{Introduction}

Nowadays, many wireless electronic devices could be used to exchange data between different points on, inside or at the proximity of the human body. Data 
could be medical related, e.g., electrocardiogram (ECG), electroencephalogram (EEG), electromyogram (EMG), etc., or consumer lifestyle related, e.g., photos, videos, messages, etc. Nevertheless, airborne transmission using antennas typically is a very energy intensive process implying large batteries. Therefore, low power consumption with high quality transmission techniques are highly needed. Human body communication (HBC) is one transmission technique defined for low power consumption in wireless body area networks (WBANs). Different ways can be used to establish BAN communication networks [1]. In this paper, we focus on HBC based on galvanic coupling approach. Here, the human body is considered as the transmission medium for data signals. Therefore, the signals are confined to the body, which ensures high security and less interference with neighboring transceivers during signal transmission. Moreover, HBC is a promising technique for low power consumption transmission, which helps the batteries of wearable or implantable devices to be reliable and longer lasting. In addition, harvesting energy from the human body could be used to continuously recharge HBC-enabled devices without the need for external power supplies [2].

HBC transmitter (TX) and receiver (RX) are each connected to a coupler that is composed of two electrodes, i.e., signal electrode and ground. In the galvanic coupling, digital information is exchanged by inducing weak AC currents galvanically into the human body. Both TX and RX electrodes are hence attached directly to the body.

For applications of $\mathrm{HBC}$, two possible communication connections can be established. In intra-body communication, data is transferred between sensors distributed across the same human body, which enables data fusion, e.g. for monitoring of health condition. In contrast, in inter-body communication, data is transferred between sensors distributed across different bodies that are in touch. This will allow users to be able to exchange data among several wearable devices just by touching each other [3]. One application is shown in Fig. 1: Different sensors are distributed across the human body of a patient, which collect his vital signs, e.g., EMG, ECG, (electrooculogram) EOG, etc., then send this data to a central processing unit (CPU) to be combined, encoded and further resent to an HBC-enabled device worn by a doctor for data decoding and displaying.

Several studies have been previously done to investigate transmission characteristics of intra-body communication either with experimental studies or with simulations [4-12]. In [4], an experimental study was done to send data through the tissues from an implanted telemetry device to be detected by surface electrodes. In vivo experiments were conducted in [5] to analyze the human data channel. The authors in [6] have developed a wearable ECG for vital signals transmission based on impulse radio type HBC. In addition, numerical simulations and experiments were conducted in [7] to understand the influences from the electrode size, joints between TX and RX, and electrode positions on signal attenuation. In [8], the authors proposed a transfer function to represent the galvanic coupling in intra-body communication, moreover, some in vivo measurements were conducted. Also, a simulated-based model of the whole human body was introduced to measure the signal attenuation at different positions 


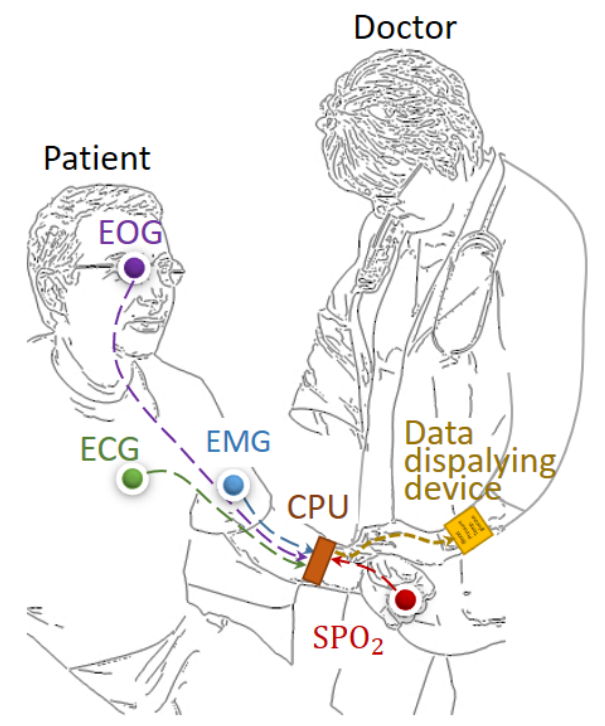

Fig. 1: Potential application scenario for human body communication: Vital data from several sensors are transmitted to a central hub and processing unit (intra-body communication); relevant data is then sent to the treating physician (inter-body communication).

on the body. In [9], several types of electrodes are compared and signal attenuation between different positions on the human body was investigated via in vivo measurements. In [10], the authors proposed a 3D FEM multilayered cylindrical model of a human arm to simulate the current density and electric field through different tissues as a function of frequency for different channel lengths and inter-electrode distances. In [11,12], we have tried to understand the transmission mechanism in intra-body communication based on a simulated model of the arm, the electric potential difference at TX and RX at different applied frequencies, the current density, inter-electrode distances and the signal attenuation on the surface of the body and inside tissues were investigated.

In this paper, the data transmission mechanism in the human tissues for both intra-body and inter-body communication is investigated, which to the best of the authors' knowledge, has not been enough covered in the HBC literature. Two different simulation-based models are implemented using COMSOL Multiphysics 5.3a software. In a model of a human arm consisting of different types of tissues, the transmission mechanism in intra-body communication in a straight simulated-based arm model is studied, in addition, some bending is introduced to the model to investigate its effect on signal attenuation. On the other hand, with the model of two contiguous human arms, signal transmission in inter-body communication while changing the area of contact between the arms is investigated. 
This paper is organized as follows. In section 2, we derived dielectric properties of the human tissues used in our simulation; in addition, some theoretical framework and approximations are given. In section 3, the simulation setup and results are given. Finally, section 4 concludes our work.

\section{Dielectric Properties of Human Tissues and Approximations}

The dielectric properties of body tissues at various frequencies are represented in graphical plots using a model based on the summation of 4-Cole-Cole expressions, which describes the complex relative permittivity as follow [13]:

$$
\epsilon_{r}(\omega)=\epsilon_{\infty}+\sum_{m=1}^{4} \frac{\Delta \epsilon_{m}}{1+\left(j \omega \tau_{m}\right)^{1-\alpha_{m}}}+\frac{\sigma_{i}}{j \omega \epsilon_{0}}
$$

where $\epsilon_{r}(\omega)$ represents the frequency-dependent complex relative permittivity, $\epsilon_{\infty}$ is the material permittivity at very high frequencies, $\Delta \epsilon_{m}, \tau_{m}$, and $\alpha_{m}$ are material parameters, $\sigma_{i}$ is the ionic conductivity, $j$ denotes the imaginary unit $\sqrt{-1}, \omega$ is the angular frequency, and $\epsilon_{0}$ the permittivity of free space. Table 1 shows the parameters needed to find the complex relative permittivity of the human tissues used in our models [14]. From Eq. (1) with Table 1, we derived the required dielectric properties for our simulation.

Due to [15], quasi-static approximation can be applied if the dimensions of the system are small compared to the wavelength of the applied signal. In our study, we used electrical signals with frequencies up to $1 \mathrm{MHz}$, which means

Table 1: Parameters for the derivation of dielectric properties of different human tissues. (Adapted from [14])

\begin{tabular}{lccccc}
\hline Tissue & Skin $($ dry $)$ & Fat & Muscle & cortical & cancellous \\
\hline$\epsilon_{\infty}$ & 4.00 & 2.50 & 4.00 & 2.50 & 2.50 \\
$\Delta \epsilon_{1}$ & 32 & 9 & 50 & 10 & 18 \\
$\tau_{1}(p s)$ & 7.234 & 7.958 & 7.234 & 13.263 & 13.263 \\
$\alpha_{1}$ & 0.000 & 0.200 & 0.100 & 0.200 & 0.220 \\
$\Delta \epsilon_{2}$ & 1100 & 35 & 7000 & 180 & 300 \\
$\tau_{2}(n s)$ & 32.481 & 15.915 & 353.678 & 79.577 & 79.577 \\
$\alpha_{2}$ & 0.200 & 0.100 & 0.100 & 0.200 & 0.250 \\
$\sigma_{i}$ & 0.000 & 0.035 & 0.200 & 0.020 & 0.070 \\
$\Delta \epsilon_{3}$ & $0.00 \mathrm{E}+0$ & $3.30 \mathrm{E}+4$ & $1.20 \mathrm{E}+6$ & $5.00 \mathrm{E}+3$ & $2.00 \mathrm{E}+4$ \\
$\tau_{3}(\mu s)$ & 159.155 & 159.155 & 318.310 & 159.155 & 159.155 \\
$\alpha_{3}$ & 0.200 & 0.050 & 0.100 & 0.200 & 0.200 \\
$\Delta \epsilon_{4}$ & $0.00 \mathrm{E}+0$ & $1.00 \mathrm{E}+7$ & $2.50 \mathrm{E}+7$ & $1.00 \mathrm{E}+5$ & $2.00 \mathrm{E}+7$ \\
$\tau_{4}(m s)$ & 15.915 & 15.915 & 2.274 & 15.915 & 15.915 \\
$\alpha_{4}$ & 0.200 & 0.010 & 0.000 & 0.000 & 0.000 \\
\hline
\end{tabular}


that the operating wavelength is large enough compared to the dimension of the human body. Therefore, the electro-quasi-static approximation can be applied and Maxwell's equation can be simplified to the Laplace equation

$$
-\nabla \cdot\left(\left(\epsilon+\frac{\sigma}{j \omega}\right) \nabla V\right)=0
$$

where $\sigma$ is the conductivity and $V$ represents the scalar electric potential.

\section{Simulation-based Human Arm Models}

\subsection{Simulation Setup}

Two finite element method (FEM) models were implemented using COMSOL Multiphysics 5.3a. In order to investigate intra-body communication, the complex structure of the human arm was approximated by a five-layers concentric cylinder of radius $3.5 \mathrm{~cm}$ and length $60 \mathrm{~cm}$. The layers are skin, fat, muscle, cortical bone, and cancellous bone of thicknesses $0.126,0.58,1.55,0.6$ and $0.644 \mathrm{~cm}$, respectively. These values were chosen to be within the anatomical range [16-19]. For inter-body communication, on the other hand, a second contiguous similar cylinder was added, where the cylinders represent two human arms that touch each other. For simplicity, the single arm model connected to the TX was termed "Arm 1 " and the second contiguous arm was called "Arm 2 ". The dielectric properties of the human tissues used in our models at different frequencies were derived as described in section 2 and applied in COMSOL using interpolation functions. The tissues were defined as homogeneous frequency dependent materials. Extra-fine mesh size was applied to the geometry. The domain around the geometry was chosen to be air that extends to infinity. The two TX and two RX electrodes are in direct contact to the human body. In our work, the electrodes were assumed to be circular and made of copper with radius $1 \mathrm{~cm}$ and thickness $0.2 \mathrm{~cm}$. The electrical parameters of the copper material are already defined in COMSOL. An inner-distance of $l_{i}=4.5 \mathrm{~cm}$ was taken between TX or RX electrodes. A current controlled waveform with different amplitudes, i.e., 1 and $4 \mathrm{~mA}$, was applied that meet the safety standardization according to [20]. The current amplitudes are galvanically coupled into the arm model through the TX signal electrode with a frequency sweep from $10 \mathrm{kHz}$ up to $1 \mathrm{MHz}$. This frequency range was chosen to be above the frequencies of the biological signals and still fulfills the quasi-static approximation. The main parameters used in the simulation are summarized in Table 2.

\subsection{Model for Intra-body Communication}

Model Geometry and setup In the intra-body communication model, two studies have been conducted. In one study, a straight arm model, i.e., Arm 1 , has been used. In a second study, a $90^{\circ}$ bending has been introduced to the model to imitate the elbow bending and investigate its effect on the received signal. Transmitting and receiving electrodes were placed symmetrically around the curvature of the bended arm as seen in Fig. 2. 


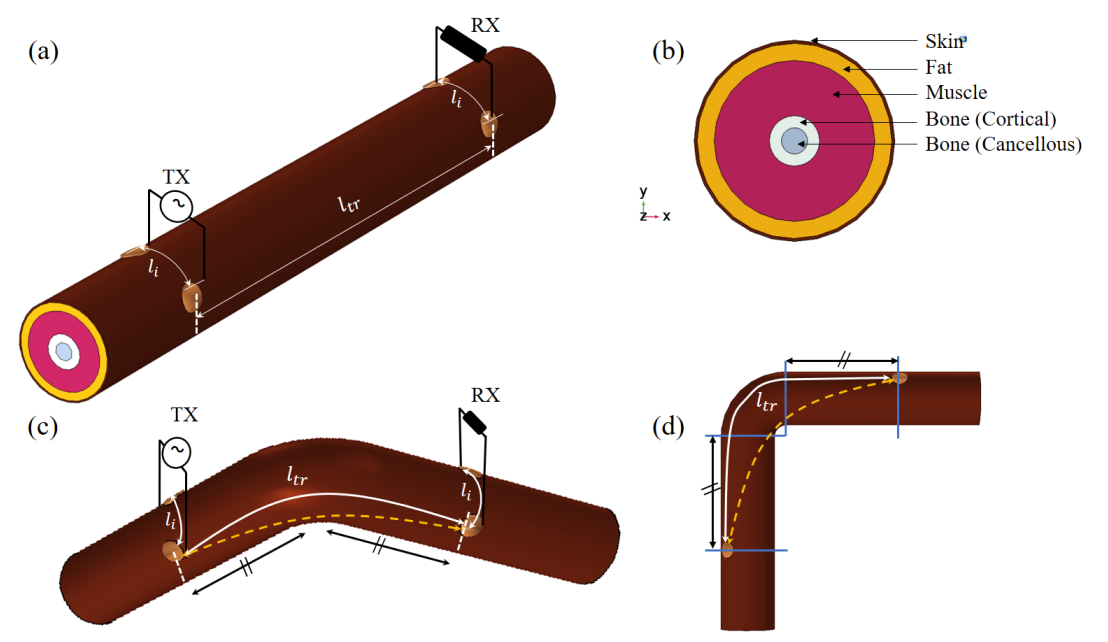

Fig. 2: Simulation-based model of a single human arm: (a) 3D straight model, (b) crosssectional view, (c) 3D model with $90^{\circ}$ bending, (d) side view of the bended model.

Results and Discussion Fig. 3(a) shows the electrical potential distribution for a cross sectional area of the arm model at TX at an induced current of $1 \mathrm{~mA}$, and applied frequency $10 \mathrm{kHz}$. The same setup but at applied frequency of $1 \mathrm{MHz}$ is shown in Fig. 3(b). In Fig. 3(c), we changed the induced current to $4 \mathrm{~mA}$ with $10 \mathrm{kHz}$ applied frequency. In Fig. 3(d), $4 \mathrm{~mA}$ induced current with frequency of $1 \mathrm{MHz}$ was applied. Regardless of the induced current, the electric potential decreases with the increase of the applied frequency. At the lower frequency, i.e., $10 \mathrm{kHz}$, the relative permittivity of the human tissues is high and conductivity is low. Therefore, penetration of the signal into the tissue is high. As seen, the electric potential is mainly confined to the skin and fat layers, while less potential exists in the muscle and bones layers. At the higher frequency, i.e., $1 \mathrm{MHz}$, in contrast, when the relative permittivity is low and conductivity is high, signal transmission is limited to the surface of the body. However, increasing the amplitude of the induced current at a fixed applied frequency has a significant effect on the generated signal. Nevertheless, we should follow the human safety standardization for not harming the biological tissues.

In Fig. 4(a), we studied the effect of increasing the surface distance between TX and RX, i.e., $l_{t r}$, when keeping constant current of $1 \mathrm{~mA}$, while in Fig. 4(b), the signal attenuation is shown. The attenuation factor between TX and RX was calculated according to [7]

$$
\text { Attenuation }[\mathrm{dB}]=20 \cdot \log _{10} \frac{\Delta V_{\mathrm{RX}}}{\Delta V_{\mathrm{TX}}}
$$

where $\Delta V_{\mathrm{RX}}$ and $\Delta V_{\mathrm{TX}}$ denote the potential difference at the receiver and transmitter electrodes, respectively. At low frequencies, the increase of $l_{t r}$ has a weak effect on the received signal. This gives a good opportunity to use intra-body 
Table 2: Parameters for the simulation-based model.

\begin{tabular}{l|l}
\hline Parameters & value \\
\hline Frequency range " $f$ " $[\mathrm{Hz}]$ & $10^{4}-10^{6}$ \\
Length of the Arm $[\mathrm{cm}]$ & 60 \\
Skin layer thickness $[\mathrm{cm}]$ & 0.126 \\
Fat layer thickness $[\mathrm{cm}]$ & 0.58 \\
Muscle layer thickness $[\mathrm{cm}]$ & 1.55 \\
Cortical bone thickness $[\mathrm{cm}]$ & 0.6 \\
Cancellous bone thickness $[\mathrm{cm}]$ & 0.644 \\
Current amplitude "I" [mA] & 1,4 \\
Radius of copper electrodes $[\mathrm{cm}]$ & 1 \\
Thickness of copper electrodes $[\mathrm{cm}]$ & 0.2 \\
Distance between TX and RX " $l_{t r} "[\mathrm{~cm}]$ & $20,30,40,50$ \\
Inner-distance between TX or RX electrodes $" l_{i} "[\mathrm{~cm}]$ & 4.5 \\
\hline
\end{tabular}

(1ea(1)=10000 Hz Suface: Electric potential (v)

(a)
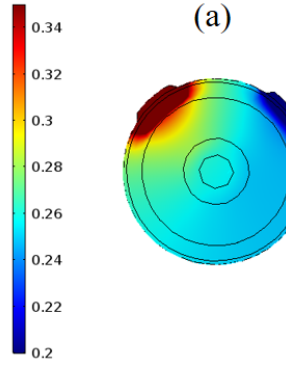

req(1) $=10000 \mathrm{~Hz}$ Surface: Electric potential (V)

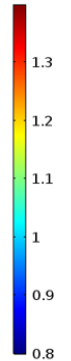

freq(50) $=1 \mathrm{E} 6 \mathrm{~Hz}$ Surface: Electric potential $(\mathrm{V})$

(b)

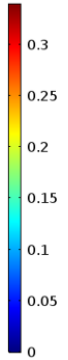

freq(50) $=1 \mathrm{E} 6 \mathrm{~Hz}$ Surface: Electric potential (V)

(d)

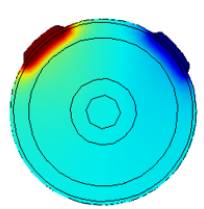

Fig. 3: Electric potential distribution for cross sectional area of the straight arm model at TX when: (a) $I=1 \mathrm{~mA}, f=10 \mathrm{kHz}$, (b) $I=1 \mathrm{~mA}, f=1 \mathrm{MHz}$, (c) $\mathrm{I}=4 \mathrm{~mA}, f$ $=10 \mathrm{kHz}$, and $(\mathrm{d}) \mathrm{I}=4 \mathrm{~mA}, f=1 \mathrm{MHz}$. 

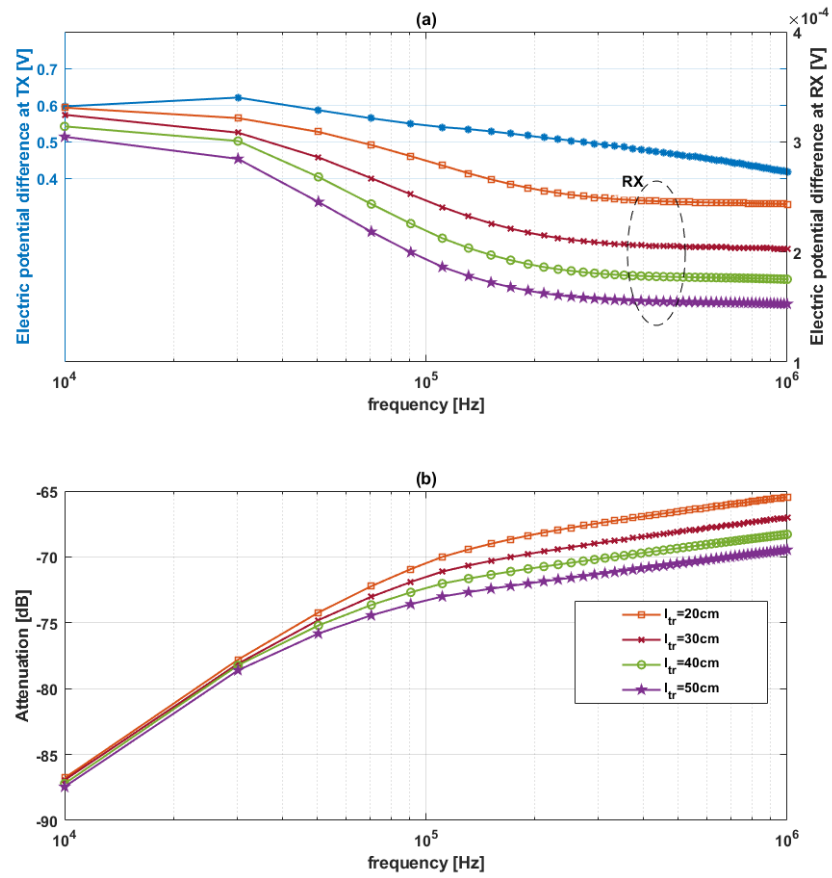

Fig. 4: (a) Electrical potential difference at TX and RX at $I=1 \mathrm{~mA}$, when $l_{t r}=20 \mathrm{~cm}$, $l_{t r}=30 \mathrm{~cm}, l_{t r}=40 \mathrm{~cm}$, and $l_{t r}=50 \mathrm{~cm}$, (b) Signal attenuation $[\mathrm{dB}]$ at RX for different channel lengths.

communication at low frequencies, e.g., up to $50 \mathrm{kHz}$, for sending data between distant locations on the body. However, frequencies above the biological signals frequencies should be used to avoid interference. On the other hand, a significant effect can be seen at higher frequencies, where the signal loss inside the tissues is high. Therefore, the attenuation increases, which agrees with the results in [7]. In another study, to investigate the effect of $90^{\circ}$ bending of the arm model and compare it to the straight model, we calculated the electric potential difference at RX for both cases when the induced current is $1 \mathrm{~mA}$ for different $l_{t r}$. As seen in Fig. 5, the electric potential difference at RX of the bended model is higher than that of the straight model. The reason is the actual channel length or the shortest distance between TX and RX. In the straight model, the channel length equals the direct horizontal distance from TX to RX. In the bended model, the actual channel length, shown as dashed yellow line in Fig. 2, is shorter than the surface length we considered. At short distances between TX and RX, the relative discrepancy between the actual channel length in the straight model and the bended model is large. On the other hand, for longer models and distant transceivers around the arm curvature, the relative discrepancy becomes small and both models tend to behave in a close manner, see Fig. 5 when $l_{t r}=40 \mathrm{~cm}$, and $50 \mathrm{~cm}$. 


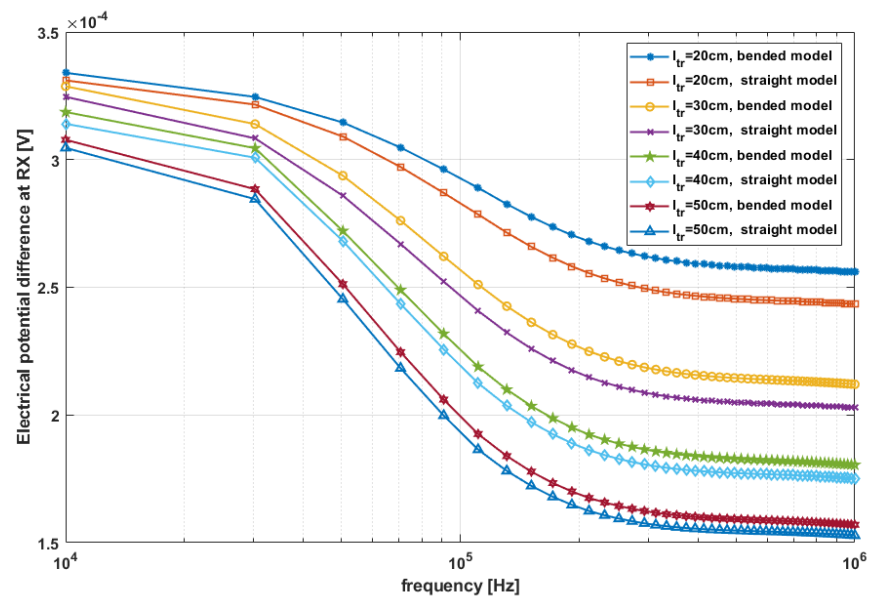

Fig. 5: Electrical potential difference at RX for both bended and straight models at $I$ $=1 \mathrm{~mA}$.

\subsection{Model for Inter-body Communication}

Model Geometry and Setup In the inter-body communication model, the second contiguous similar cylinder "Arm 2 " was added, as shown in Fig. 6. Different common lengths $l_{c}=5,10$, and $20 \mathrm{~cm}$ between $A_{1 r m}$ and $A_{2 r m}$ were assumed. A contact plate of width $2 \mathrm{~cm}$ and length $l_{c}$ was added between the two arms to increase their contact area and defined in COMSOL as skin material, shown in Fig. 6 inside dotted blue ellipsoids.

Results and Discussion Fig. 7 shows the electric potential difference at RX for a horizontal distance $l_{t r}=20 \mathrm{~cm}$ between TX and RX and common length $l_{c}=20 \mathrm{~cm}$ at two different induced current amplitudes, i.e., $1 \mathrm{~mA}$, and $4 \mathrm{~mA}$. As expected, the electric potential is directly proportional to the amplitude of the induced current. Maximum value is achieved at $10 \mathrm{kHz}$, then the signal decreases rapidly with frequency.

For a comparison between inter-body and intra-body communication transmission mechanisms, we have created Fig. 8(a) at three different common lengths, i.e., $l_{c}=5,10$, and $20 \mathrm{~cm}$, between $\mathrm{Arm}_{1}$ and $\mathrm{Arm}_{2}$ when the current amplitude is $1 \mathrm{~mA}$ and $l_{t r}=20 \mathrm{~cm}$, while the signal attenuation from (3) is depicted in Fig. 8(b). We can see the same behavior with frequency as in intra-body communication, the potential difference decreases with the frequency increment. In addition, increasing the length of contact, i.e., $l_{c}$, and hence the contact area between the two arms has a significant effect on the detected signal. By increasing the contact area between the two arms, the detected signal increases. As seen at frequencies above $200 \mathrm{kHz}$ when $l_{c}=l_{t r}=20 \mathrm{~cm}$, intra-body and inter-body communication give close values. Such that the two arms together can be consid- 


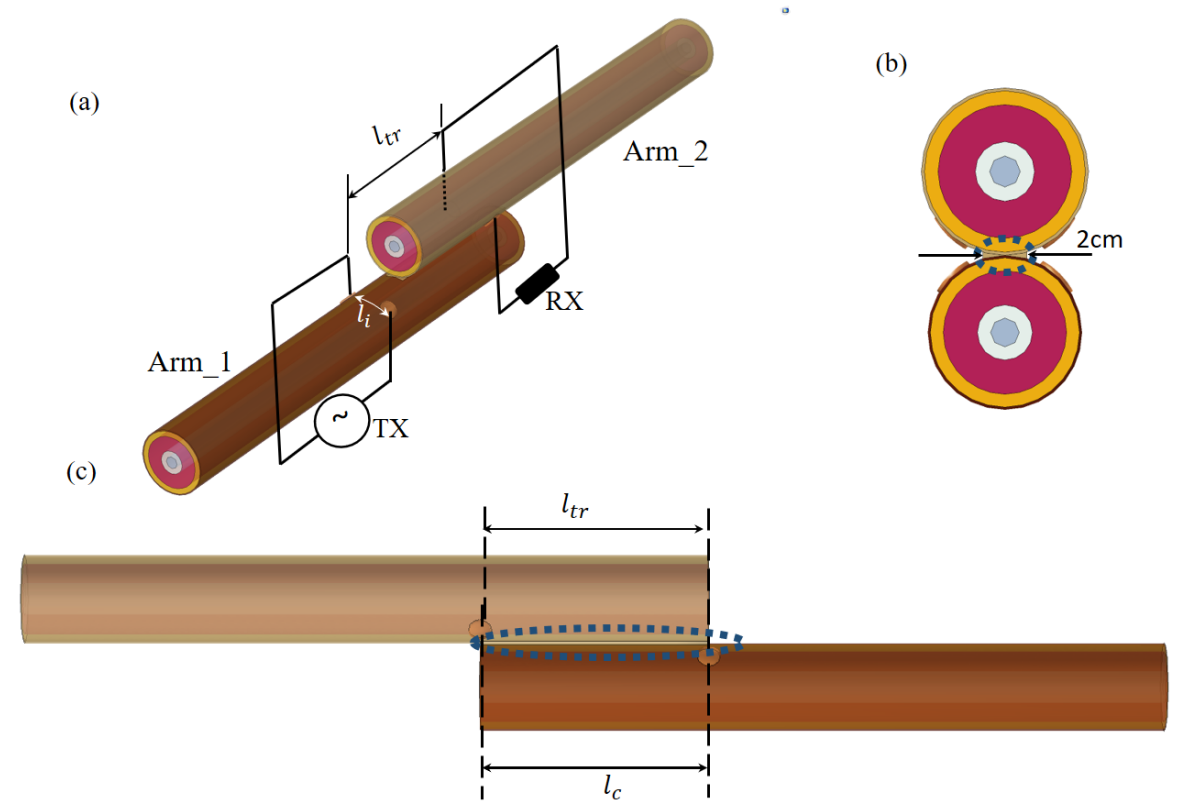

Fig. 6: Simulation-based model of two contiguous human arms. (a) 3D model, (b) cross sectional view, (c) side view.

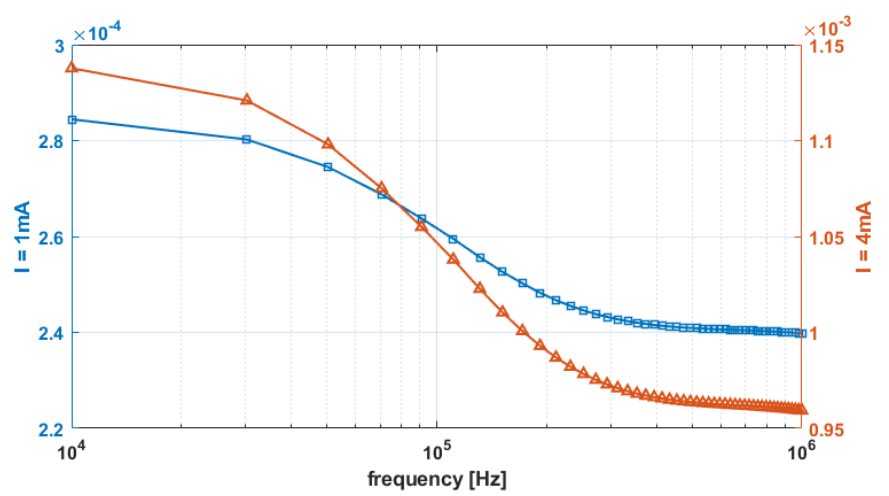

Fig. 7: Electric potential difference at RX for $l_{c}=20 \mathrm{~cm}$ and $l_{t r}=20 \mathrm{~cm}$ with two different induced current amplitudes.

ered as one geometry that uses intra-body communication. For shorter $l_{c}$, high signal degradation is detected at the RX at $\mathrm{Arm}_{2}$. Hence, one factor to improve the received signal in inter-body communication is to avoid gaps and increase the contact area between the two bodies. However, inter-body communication might be power consuming communication technique and hence adaptive HBC-enabled devices are needed. 

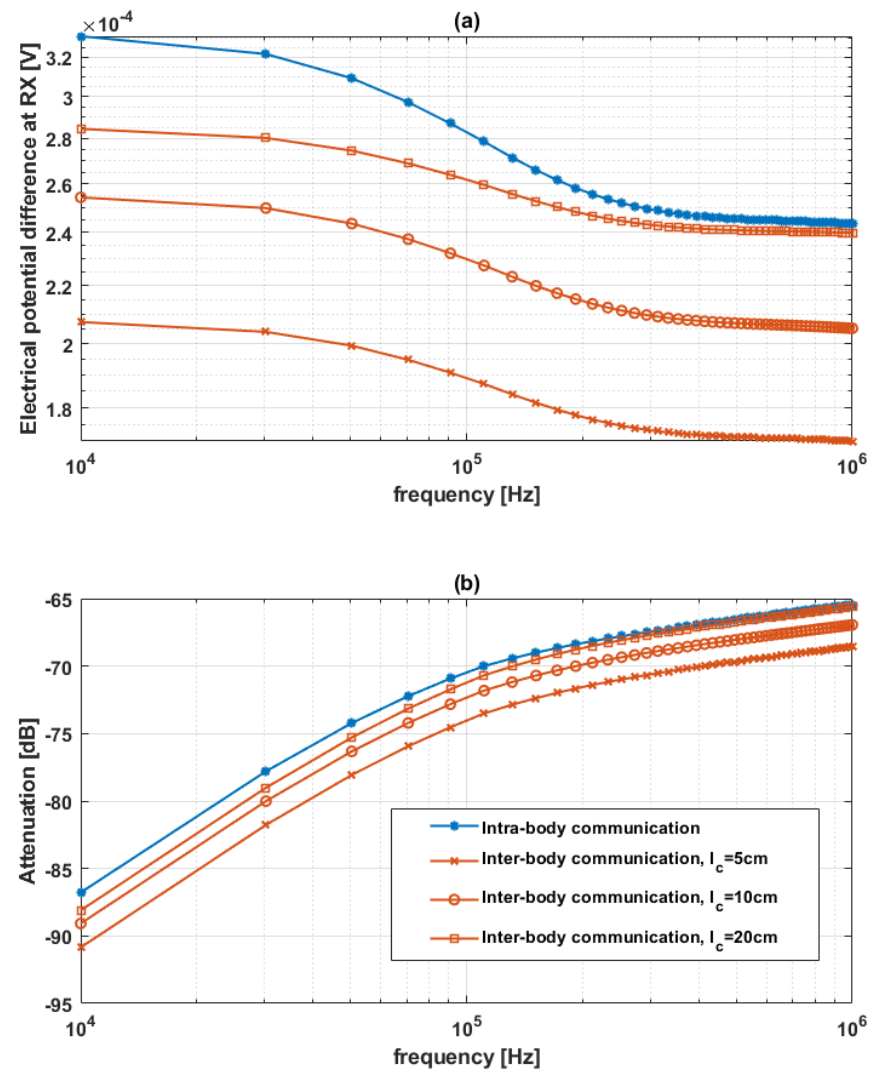

Fig. 8: Electric potential difference at RX for both intra-body and inter-body communications with three different $l_{c}$ values at an induced current of $1 \mathrm{~mA}$ and $t_{t r}=20 \mathrm{~cm}$, (b) Signal attenuation $[\mathrm{dB}]$ at RX.

\section{Conclusion}

Galvanic coupling is one of the approaches used for coupling in human body communication (HBC), which enables electronic devices in or on the human body to interconnect with each other and exchange information at very low power consumption. In this paper, we have studied the transmission mechanism on the human body for intra-body and inter-body communication based on galvanic coupling. Two FEM simulations representing a single human arm and two human arms in contact to each other have been proposed. Both models took into account the different types of tissue that the arm consists of. The influence of different HBC key parameters including applied frequency, distance between TX and RX (i. e., transmission length), bending, contact area between two bodies, and induced current were investigated. The results show that the transmission loss increases with the increase of the transmission length and operating fre- 
quency. The electric potential is mainly confined to the skin and fat layers at lower frequencies, as the relative permittivity of the human tissues is high and conductivity is low. At high frequencies, when the relative permittivity is low and conductivity is high, the transmitted signal is limited to the surface of the body. Furthermore, increasing the transmission length has a significant effect on the signal degradation at high frequencies, hence it is more appropriate to use low frequencies, i.e., up to $50 \mathrm{kHz}$, for transmission over long distances on the body. Bended models improve the received signal in the cases of short distance between TX and RX around the bending. For distant transceivers, both straight and bended models tend to behave in a close manner.

Concerning inter-body communication, we found that the received signal is directly proportional to the induced current and inversely proportional to the applied frequency. Due to the dielectric properties of the human tissues and the increase of the actual transmission distance between TX and RX (in order for the signal to leave one arm and enters the other), the signal degradation is considerably higher compared to intra-body communication at the same horizontal distance between TX and RX. At higher frequencies, i.e., $>200 \mathrm{kHz}$, both inter-body and intra-body communication give close values when the contact area between the arms covers the distance between TX and RX electrodes. Increasing the contact area between the models helps to improve the detected signal.

In conclusion, the results demonstrate the feasability of HBC for establishing wireless communication between distributed sensor nodes in or on the human body as well as the exchange of information between different individuals. The approach hence promises diverse ways application, e.g., in healthcare and telecommunications.

\section{References}

1. Nikita, K. S.: Numerical and Experimental Techniques for Body Area Electromagnetics. Handbook of Biomedical Telemetry.1st edn., John Wiley \& Sons, USA(2014).https://doi.org/10.1002/9781118893715.ch6

2. Johny, B., Anpalagan, A.: Body Area Sensor Networks: Requirements, Operations, and Challenges. IEEE Potentials, vol. 33, 21-25 (2014).https://doi.org/10.1109/MPOT.2013.2286692

3. Ruiz J. A., Shimamoto, S.: A study on the transmission characteristics of the human body towards broadband intra-body communications. Proceedings of the Ninth International Symposium on Consumer Electronics, 99-104 (2005).https://doi.org/10.1109/ISCE.2005.1502350

4. Lindsey, D.P., McKee, E.L., Hull, M.L., Howell, S.M.: A new technique for transmission of signals from implantable transducers. IEEE Transactions on Biomedical Engineering, vol. 45, 614-619 (1998).https://doi.org/10.1109/10.668752

5. Gao, Y., Ma, R., Pun, S. Mak, P., Vai, M.: Measurement system with experiments for galvanic coupling type intra-body communication. 5th International Conference on Biomedical Engineering and Informatics, 761-764 (2012).https://doi.org/10.1109/BMEI.2012.6513147 
6. Wang, J. , Fujiwara, T., Kato, T., Anzai, D.: Wearable ECG Based on Impulse Radio Type Human Body Communication. IEEE Transactions on Biomedical Engineering, vol. 63, 1887-1894 (2016).https://doi.org/10.1109/TBME.2015.2504998

7. Wegmueller, M. S., Kuhn, A., Froehlich, J., Oberle, M., Felber, N., Kuster, N., Fichtner, W.: An Attempt to Model the Human Body as a Communication Channel. IEEE TransactionsON Biomedical Engineering, vol. 54, no. 10, 18511857(2007).https://doi.org/10.1109/TBME.2007.893498

8. Song,Y., Hao, Q., Zhang, K.: Review of the Modeling, Simulation and Implement of Intra-body Communication. ScienceDirect. Defence Technology 9, 10-17 (2013).https://doi.org/10.1016/j.dt.2013.10.001

9. Wegmueller, M. S., Oberle, M., Felber, N., Kuster, N., Fichtner, W.: Signal Transmission by Galvanic Coupling Through the Human Body. IEEE Transactions on Instrumentation and Measurement, vol. 59, no. 4, 963969(2010).https://doi.org/10.1109/TIM.2009.2031449

10. Callejón, M. A., et al.: Galvanic Coupling Transmission in Intrabody Communication: A Finite Element Approach. IEEE Transactions on Biomedical Engineering, vol. 61, 775-782 (2014).https://doi.org/10.1109/TBME.2013.2289946

11. Ahmed, D., Kirchner, J., Fischer, G.: Wave propagation with human body communications in BANs. IEEE MTT-S International Conference on Numerical Electromagnetic and Multiphysics Modeling and Optimization for RF, Microwave, and Terahertz Applications, 16-18 (2017).https://doi.org/10.1109/NEMO.2017.7964172

12. Ahmed, D., Kirchner, J., Fischer, G.: Wave Propagation with HBC in a Human Arm Model. IEEE International Symposium on Medical Measurements and Applications (MeMeA), (2017).https://doi.org/10.1109/MeMeA.2017.7985918

13. Gabriel, S., Lau, R. W., Gabriel, C.: The dielectric properties of biological tissues: II. Measurements in the frequency range $10 \mathrm{~Hz}$ to $20 \mathrm{GHz}$. Physics in Medicine and Biology 41, 2251-2269 (1996). https://doi.org/10.1088/0031-9155/41/11/002

14. Hall, P. S., Hao, Y.: Electromagnetic Properties and Modeling of the Human Body. Antennas and Propagation for Body-Centric Wireless Communications. 2nd edn. Artech House, ch.2, London (2012).

15. larsson, J.: Electromagnetics from a quasistatic perspective. American Journal of Physics 75 (3), 230-239 (2007).https://doi.org/10.1119/1.2397095

16. Wilkinson, P. F., Millington, R.: Skin (Digitally printed version edn.). Cambridge University Press, 49-50 (2009).

17. Hwang, I. D., Shin, K.: Fat thickness measurement using optical technique with miniaturized chip LEDs : A preliminary human study. Proceedings of the 29th Annual International Conference of the IEEE EMBS, 4548-4551 (2007).https://doi.org/10.1109/IEMBS.2007.4353351

18. Dupont, A., et al.: Real-Time Sonography to Estimate Muscle Thickness: Comparison with MRI and CT. Journal of Clinical Ultrasound, 230-236 (2000).https://doi.org/10.1002/jcu.1025

19. Horsman, A., Leach, A. E.: The Estimation of the Cross-sectional Area of the Ulna and Radius. American Journal of Physical Anthropology, 173-186 (1974).https://doi.org/10.1002/ajpa.1330400204

20. International Commission on Non-Ionizing Radiation Protection. Guidelines for Limiting Exposure to Time-Varying Electric, Magnetic, and Electromagnetic Fields (up to $300 \mathrm{GHz}$ ). Health Physics 74(4), 494-522 (1998). 